\title{
Skin Sensitisation: The Colipa Strategy for Developing and Evaluating Non-Animal Test Methods for Risk Assessment
}

\author{
Gavin Maxwell ${ }^{1}$, Pierre Aeby ${ }^{2}$, Takao Ashikaga ${ }^{3}$, Sandrine Bessou-Touya ${ }^{4}$, Walter Diembeck ${ }^{5}$, \\ Frank Gerberick ${ }^{6}$, Petra Kern ${ }^{6}$, Monique Marrec-Fairley ${ }^{2}$, Jean-Marc Ovigne ${ }^{7}$, \\ Hitoshi Sakaguchi ${ }^{8}$, Klaus Schroeder ${ }^{9}$, Magali Tailhardat ${ }^{10}$, Silvia Teissier ${ }^{7}$ and Petra Winkler ${ }^{11}$ \\ ${ }^{1}$ Unilever, Sharnbrook, UK; ${ }^{2}$ Colipa, Brussels, Belgium; ${ }^{3}$ Shiseido Research Centre, Yokohama-Shi, Japan; ${ }^{4}$ Pierre Fabre, \\ Castres, France; ${ }^{5}$ Beiersdorf, Hamburg, Germany; ${ }^{6}$ The Procter \& Gamble Company, Cincinnati, OH, USA and Strombeek- \\ Bever, Belgium; ${ }^{7}$ L'Oréal, Aulnay sous Bois, France; ${ }^{8}$ Kao Corporation, Tochigi, Japan; ${ }^{9}$ Henkel AG \& Co, Düsseldorf, \\ Germany; ${ }^{10}$ LVMH Recherche, Saint Jean de Braye, France; ${ }^{11}$ Johnson \& Johnson, Neuss, Germany
}

\begin{abstract}
Summary
Allergic contact dermatitis is a delayed-type hypersensitivity reaction induced by small reactive chemicals (haptens). Currently, the sensitising potential and potency of new chemicals is usually characterised using data generated via animal studies, such as the local lymph node assay (LLNA). There are, however, increasing public and political concerns regarding the use of animals for the testing of new chemicals. Consequently, the development of in vitro, in chemico or in silico models for predicting the sensitising potential and/or potency of new chemicals is receiving widespread interest.

The Colipa Skin Tolerance task force currently collaborates with and/or funds several academic research groups to expand our understanding of the molecular and cellular events occurring during the acquisition of skin sensitisation. Knowledge gained from this research is being used to support the development and evaluation of novel alternative approaches for the identification and characterisation of skin sensitising chemicals. At present three non-animal test methods (Direct Peptide Reactivity Assay (DPRA), Myeloid U937 Skin Sensitisation Test (MUSST) and human Cell Line Activation Test (hCLAT)) have been evaluated in Colipa interlaboratory ring trials for their potential to predict skin sensitisation potential and were recently submitted to ECVAM for formal pre-validation. Data from all three test methods will now be used to support the study and development of testing strategy approaches for skin sensitiser potency prediction.

This publication represents the current viewpoint of the cosmetics industry on the feasibility of replacing the need for animal test data for informing skin sensitisation risk assessment decisions.
\end{abstract}

Keywords: skin sensitisation, allergic contact dermatitis, animal alternatives, replacement, risk assessment

\section{Introduction}

Allergic contact dermatitis (ACD) is a delayed-type hypersensitivity reaction induced by small reactive chemicals (haptens) that can result from the induction of skin sensitisation (Smith and Hotchkiss, 2001). Guinea pig models have historically been used to identify whether a chemical has the potential to induce skin sensitisation in humans. More recently, a refined and reduced method, i.e. the murine local lymph node assay (LLNA), has been employed (Basketter et al., 2002). Sensitiser potency information generated using the LLNA can be used to predict a safe level of human exposure (using a Quantitative Risk Assessment (QRA) approach (Felter et al., 2002, 2003)). There are, however, increasing public and political concerns regarding the use of animal testing for the hazard characterisation of new chemicals, and consequently the development of in vitro, in chemico or in silico models for the replacement of animal testing is a high priority (EC, 2003; US National Research Council, 2007).

The current replacement rationale for skin sensitisation is that data from several non-animal test methods will need to be combined as part of a testing strategy to produce useful skin sensitiser potency information (Jowsey et al., 2006; Maxwell et al., 2008; Natsch et al., 2009). Each non-animal test method aims to model a key pathway involved in the induction of skin sensitisation, for example: skin exposure to the chemical and its subsequent skin bioavailability; initiation of the skin's in- 
nate immune response; chemical reactivity with skin protein; activation of epidermal (termed Langerhans cells (LC)) or dermal dendritic cells (DC); and finally, the sensitiser-specific $\mathrm{T}$ cell response (Basketter and Maxwell, 2007). Colipa (the European Cosmetics Association) currently collaborates with academic and industrial research groups in each of these key areas to increase and apply our fundamental knowledge of skin sensitisation to non-animal method development (Aeby et al., 2005, 2008; COLIPA, 2007). This publication aims to provide a progress report on all Colipa-funded research, method development and method evaluation activities, as well as providing a cosmetic industry viewpoint on the feasibility of replacement of animal testing for skin sensitisation.

\section{Skin exposure and bioavailability}

The extent and frequency of skin exposure to a sensitising chemical has long been known to play a key role in determining the potential for and severity of ACD (Basketter et al., 2006; Upadhye and Maibach, 1992). Exposure information for various product types has been generated and is routinely used to inform consumer safety risk assessments (e.g. Loretz et al., 2006, 2005, 2008). However as non-animal test methods are adopted there is an increasing need to understand skin exposure at the cellular and molecular level to enable appropriate interpretation and extrapolation of in vitro or in chemico data. Consequently, Colipa has invested in exploring the application of new technologies for the prediction of skin bioavailability and for evaluating the role of metabolism in skin sensitisation.

Toxicokinetic model for prediction of epidermal bioavailability In order to better understand the penetration and skin disposition of new test chemicals, Prof. Jerry Kasting at the University of Cincinnati, USA has developed a toxicokinetic model to allow a more accurate prediction of the epidermal bioavailability of potential skin sensitisers. This computer model of skin absorption is based on skin physico/chemical data and aims to predict key skin parameters such as; $\mathrm{C}_{\max }$ (the peak mid epidermal concentration of freely diffusing permeant); $\mathrm{AUC}_{120}$ (the area under the concentration-time profile of freely diffusing permeant 120 hours after dosing) and percentage of dose absorbed.

\section{Skin metabolism}

Understanding the metabolic competency of in vivo human skin and its role in the activation and clearance of sensitising chemicals is relevant for both the development and evaluation of in vitro test methods. Consequently Colipa is currently working with Prof. Ellen Fritsch at University of Düsseldorf, Germany and Dr Rob Edwards at Imperial College London, UK to evaluate the metabolic competency of human skin relative to in vitro human epidermal equivalents and immortalised keratinocyte models. The Imperial College project aims to profile the ecpression of metabolic enzymes in human skin means of immunochemistry and proteomics, while the University of Düsseldorf project focuses on the characterisation of basal and inducible metabolic enzyme activity. Initial results from the Imperial College group have already demonstrated that human skin may express far fewer cytochrome P450 enzymes than previously documented.

\section{Modification of skin proteins by chemical sensitisers}

Chemical sensitiser-specific $\mathrm{T}$ cells do not recognise the chemical itself but instead recognise a conjugate of the chemical (hapten) in the context of a protein fragment (a peptide), which is presented to T cells by antigen-presenting cells, such as LC or DC. In addition, chemical sensitisers can act either as a hapten (i.e. chemical is inherently reactive and will covalently bind to amino acid side chains), pro-hapten (chemical requires metabolic conversion to a protein-reactive species) or as pre-hapten (chemical conversion to a protein-reactive species). Colipa is supporting the development of next generation peptide binding test methods in addition to funding research to explore the correlation between sensitisation potential and chemical reactivity.

\section{Direct Peptide Reactivity Assay (DPRA)}

The DPRA protocol was developed by Dr. F. Gerberick at Procter \& Gamble, Cincinnati, USA in collaboration with Prof. J. P. Lepoittevin at the University of Strasbourg, France (Gerberick et al., 2007). The test method aims to model protein haptenation in chemico by measuring the depletion of two synthetic peptides (containing either a single cysteine or lysine side-chain as a reaction target) using High Performance Liquid Chromatography (HPLC). The prediction model for the DPRA uses a classification tree approach to assign different reactivity categories to the depletion data from the cysteine and lysine synthetic peptides (Gerberick et al., 2007). Using this approach the DPRA achieved $89 \%$ concordance with LLNA potency categories (i.e. weak, moderate, etc.), and following two rounds of ring trials interlaboratory reproducibility was also found to be robust. Consequently, the current protocol has been transferred to the European Centre for the Validation of Alternative Methods (ECVAM) for phase 3 pre-validation (Hartung et al., 2004).

\section{Next generation peptide binding assay}

Colipa continues to support the ongoing collaboration between Procter \& Gamble and the University of Strasbourg, which is currently focussed on increasing the applicability domain of the current test method to include poorly soluble chemicals and prohaptens that require oxidation to become active.

\section{Skin innate immune response}

Keratinocytes are the predominant epidermal cell type and are known to play a key role in the activation of LC in response to a pathogenic stimulus or physical/chemical stress (Cumberbatch et al., 1992; Griffiths et al., 2005). Although keratinocyte activation in isolation is unlikely to be directly predictive of skin sensitisation hazard, Colipa remains interested in the role that 
epidermal keratinocytes and/or dermal fibroblasts could play in the activation of LC and dermal DC. Consequently a collaboration was recently, initiated with Dr A. Ghaem Maghami at University of Nottingham, UK and Dr. J. Haycock at University of Sheffield, UK, to aims to establish an immunocompetent skin equivalent model using keratinocytes, fibroblasts and DC.

\section{Sensitiser-induced dendritic cell activation}

The next key step in the induction of the skin sensitisation process is the uptake and processing of the haptenated skin proteins by immature DCs in response to the inflammatory signals released by activated epidermal keratinocytes and dermal fibroblasts (Cumberbatch et al.,1992; Griffiths et al., 2005). During this process DCs mature to an activated state, which can be measured experimentally by assessing changes in cell surface receptor expression (e.g. co-stimulatory molecules such as CD83, CD86 or adhesion molecules such as CD54) and cytokine secretion (e.g. IL-1 $\beta$, IL-8) (Basketter and Maxwell, 2007). Advances in the generation of immature DCs in vitro from human peripheral blood monocytes and the availability of cell lines with DC-like phenotypes have led to the development of several non-animal test methods based upon the measurement of DC activation biomarkers following exposure to the test chemical.

\section{Myeloid U937 Skin Sensitisation Test (MUSST)}

Drawbacks of peripheral blood derived DCs are their complex preparation procedures, sourcing issues and their inherent donor-to-donor variability. As a possible alternative, human myeloid leukaemia cell lines represent good candidates as DC surrogates. Protocols based on the U937 cell line were developed by L'Oréal (Ade et al., 2006) and Cosmital SA, Procter \& Gamble (Python et al., 2007). The MUSST protocol is based upon the following steps (Fig. 1): U937 cells are exposed for $48 \mathrm{~h}$ in 96 well plates to a range of chemical concentrations. CD86 expression (FITC coupled monoclonal antibody) and cell viability (propidium iodide exclusion) are then measured by flow cytometry. A chemical is predicted as a sensitiser if it induces a dose-dependent increase of CD86 expression at nontoxic doses (viability $>70 \%$ ) within 2 independent experiments. Historically, the MUSST's concordance with yes/no predictions of sensitisation potential derived from human clinical data has been determined by L'Oréal to be $85 \%$ (based on 99 chemicals), and following two rounds of Colipa ring trials the inter-laboratory reproducibility of the MUSST was found to be robust. Consequently the current MUSST protocol has been transferred to ECVAM for phase 3 pre-validation.

\section{Human Cell Line Activation Test ( $h$-CLAT)}

The h-CLAT was developed in a collaboration between Kao (Sakaguchi et al., 2006) and Shiseido (Ashikaga et al., 2006). The outline of the h-CLAT protocol (Fig. 2) is as follows: THP1 cells (an acute monocytic leukaemia cell line (Tsuchiya et al., 1980)) are pre-cultured for $48 \mathrm{~h}$ or $72 \mathrm{~h}$ and are treated with the test chemical for $24 \mathrm{~h}$. After removing the test chemical, the expression of two cell surface antigens, CD86 and CD54, is measured by specific antibody staining and subsequent detection by flow cytometry. Two of three independent measurements at any dose should exceed the positive criteria (CD86 $>150 \%$ or CD54 $>200 \%$ ) in order to be judged as positive. The concordance with yes/no predictions of sensitisation potential derived from LLNA data was found to be approximately $85 \%$ (Sakaguchi et al., 2006, 2009), and following two parallel sets of ring trials involving Colipa members and Japanese cosmetic companies the h-CLAT inter-laboratory reproducibility was found to be acceptable. Consequently the current h-CLAT protocol has been

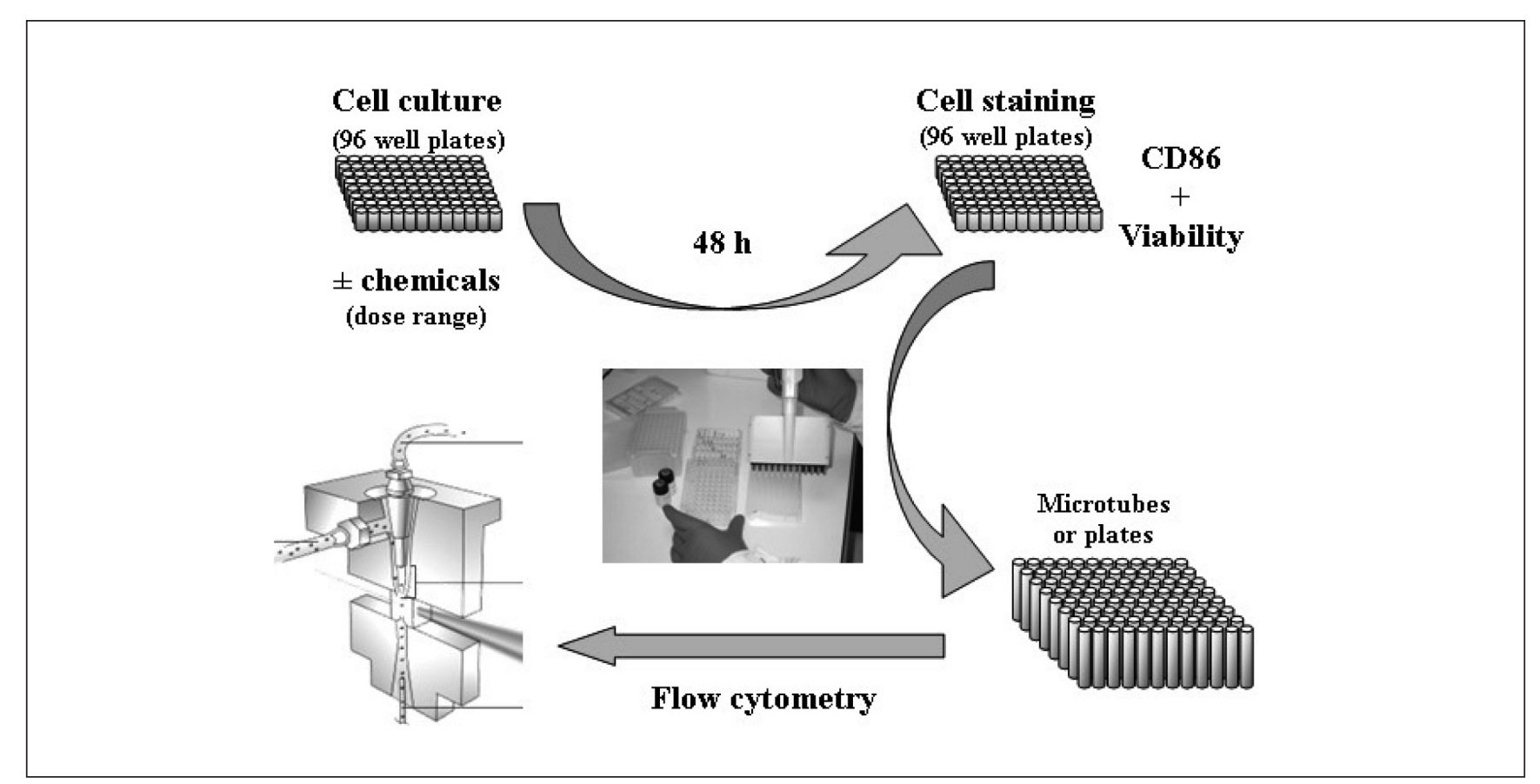

Fig. 1: Overview of MUSST protocol 
transferred to ECVAM for phase 3 pre-validation, in parallel with the MUSST and DPRA.

\section{New biomarkers for DC activation}

Intracellular signalling pathways

The early intracellular signalling pathways involved in sensitiser-induced DC activation and maturation are currently not well understood. To address this knowledge gap, Colipa recently collaborated with Prof. M. Serres at University of Claude Bernard in Lyon, France where DCs derived from human peripheral blood monocytes (MoDC), U937 and h-CLAT were treated with a large panel of sensitisers and non-sensitisers prior to ERK 1/2 and p38 MAP kinase (MAPK) activity being measured by the Face $^{\text {TM }}$ method (Trompezinski et al., 2008). The results of this study demonstrated that all sensitisers markedly activated p38 MAPK and inhibited Erk1/2 in MoDC, many of them via a predominant role of oxidative stress as previously reported. Similar data were also obtained in the U937 cell line, however the THP-1 cell line was found to respond differently, with Erk1/2 pathways becoming activated (rather than inhibited) following treatment with sensitiser. In all studies non-sensitisers had no significant impact on either p38 MAPK or Erk 1/2 kinase activity. Discussions are ongoing with respect to how best to apply these pathways as predictive biomarkers in existing or new DC activation test methods.

Gene expression changes

In order to exploit new predictive biomarkers for sensitiser-induced DC activation, Colipa has sponsored work at Procter \& Gamble (Cincinnati, USA) to develop a high throughput method for the measurement of DC gene expression changes previously identified by Affymetrix GeneChip ${ }^{\circledR}$ analysis of sensitiser-treated MoDC. This analysis revealed 173 genes that were significantly modulated (Ryan et al., 2004), and 29 of these genes were selected for further evaluation of predictive potential using RT PCR (Gildea et al., 2006). In order to develop a high throughput method the Luminex ${ }^{\circledR} \mathrm{xMAP}^{\circledR}$ technology was employed for concurrent measurement of gene expression changes, and MUTZ-3, THP-1 and U937 cell lines were all evaluated as potential surrogates for the MoDC model. All models were assessed for expression changes in all 29 genes in response to 5 sensitisers and 3 non-sensitisers, however the conclusion of the work was that, due to a lack of sensitivity, none of the cell lines was an appropriate replacement for the MoDC model.

In addition, Colipa has recently identified two projects for funding that aim to develop recombinant DC models capable of fluorescing in response to sensitiser treatment; the first project is a collaboration with Prof. A. Takashima in University of Toledo, USA that aims to develop a multi-parameter DC biosensor system for detection of skin sensitisers through transfection with a fluorescent protein or luciferase reporter construct using promoters of literature- or experimentally-identified gene biomarkers. The second project focuses on the development of a DC migration reporter model based upon coupling a luciferase construct to gene promoters known to control DC migration from the skin to the lymph nodes with Dr J. Pease at Imperial College London, UK.

\section{Sensitiser-specific $\mathbf{T}$ cell responses}

Despite sensitiser-specific $\mathrm{T}$ cell proliferation representing the hypothesised key pathway in the induction of skin sensitisation (Jowsey et al., 2006; Maxwell and MacKay, 2008), in vitro T cell

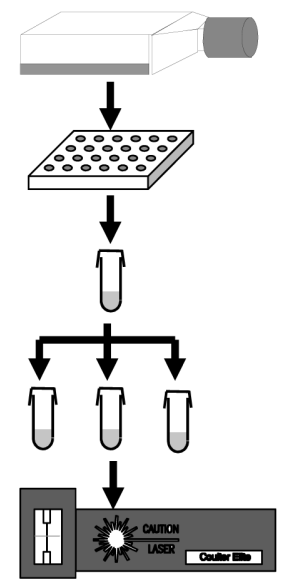

Pre-culture THP-1 cells for $48-72$ hours

Plate $\left(1 \times 10^{6}\right.$ cells/well) in 24 -well plate, treat with test chemical for 24 hours

Harvest cells, wash and block FcR $(0.01 \%$ Globulins) for $15 \mathrm{~min}$.

Divide cells into 3 aliquots, stain with FITCconjugated monoclonal antibodies (isotype control, CD86, CD54) for $30 \mathrm{~min}$.

Analyze by flow cytometry - mean

fluorescence intensity of CD86 and CD54, cell

viability by propidium iodide exclusion.

Fig. 2: Overview of h-CLAT protocol 
test methods have proven difficult to apply to chemical-induced skin sensitisation due to their low sensitivity for chemical allergens, the difficulty of ex vivo DC: T cell co-culture, the complexity of the $\mathrm{T}$ cell repertoire and the inherent genetic variability within human populations (Guironnet et al., 2000; Hauser, 1990; Rougier et al., 2000). Consequently a test method capable of generating predictive information on sensitiser-induced $\mathrm{T}$ cell responses remains a key gap at present, and Colipa is keen to identify new ways of resolving these issues.

\section{T cell priming assay (TCPA)}

For the past two years Colipa has collaborated with Prof. J. F. Nicolas at INSERM in Lyon, France to develop a human DC: $\mathrm{T}$ cell co-culture approach based on a protocol that was originally developed using murine tissues (Vocanson et al., 2006). The TCPA protocol is based around co-culturing of sensitiserhaptenated, irradiated MoDC with T cells that have been depleted for the $\mathrm{CD} 25^{+}$regulatory $\mathrm{T}$ cell subset. The rationale, which proved successful in the mouse system, is that removal of the regulatory $\mathrm{T}$ cell fraction will lower the threshold at which a $\mathrm{T}$ cell proliferative response can occur. In so doing, the sensitivity of the test method for weak and moderate sensitisers increases relative to standard DC: T cell co-culture protocols. However initial results, although demonstrating that the basic protocol can be adapted to utilise human tissues, suggest that reproducibility may still be an issue.

\section{Discussion}

To conclude, it is worth sharing the conclusions of the recent Colipa Skin Tolerance conference and Colipa "Testing Strategies for Skin Sensitisation" workshop (held on $23^{\text {rd }}-24^{\text {th }}$ June 09 and $25^{\text {th }}$ March 09, respectively), as these discussions offer perspective on the remaining challenges. For test method development, the continuing lack of a robust, reproducible in vitro method for predicting the sensitiser-specific $\mathrm{T}$ cell responses is seen as a key gap. For testing strategy development, it is felt that the lack of connectivity between different skin sensitisation biomarkers and the absence of an obvious gold standard dataset for human sensitiser potency will make establishing the clinical relevance of in vitro or in chemico biomarkers particularly challenging. In addition, the need for flexibility in testing strategy development, to accommodate new test methods and bespoke solutions for particular ingredients or exposure scenarios, represents another criterion to be accommodated. Despite these gaps, the replacement of the need for animal testing for skin sensitisation risk assessment is viewed as ultimately achievable, and the next couple of years should set the timeline for this achievement.

\section{References}

Ade, N., Martinozzi-Teissier, S., Pallardy, M. and Rousset, F. (2006). Activation of U937 cells by contact sensitizers: CD86 expression is independent of apoptosis. J. Immunotoxicol. 3, 189-197.
Aeby, P., Ashikaga, T., Diembeck, W. et al. (2008). The COLIPA Strategy for the Development of In Vitro Alternatives: Skin Sensitisation. AATEX 14, Spec. Issue, 375-379.

Aeby, P., Basketter, D. A., Diembeck, W. et al. (2005). Dendritic Cell Research Projects, Proceedings 5th World Congress 2005, ALTEX 23, Spec. Issue, 226-228.

Basketter, D. and Maxwell, G. (2007). In vitro approaches to the identification and characterization of skin sensitizers. Cutan. Ocul. Toxicol. 26, 359-373.

Basketter, D. A., Jefferies, D., Safford, B. J. et al. (2006). The impact of exposure variables on the induction of skin sensitization. Contact Dermatitis 55, 178-185.

Basketter, D. A., Evans, P., Fielder, R. J. et al. (2002). Local lymph node assay - validation, conduct and use in practice. Food and Chemical Toxicology 40, 593-598.

COLIPA (2007). Colipa, Serious About Cosmetics - Serious about Alternative Methods. http://www. colipa. com/site/index. $\mathrm{cfm}$ ?SID=15588\&OBJ=20702\&back $=1$.

Cumberbatch, M., Gould, S. J., Peters, S. W. et al. (1992). Langerhans Cells, Antigen Presentation, and the Diversity of Responses to Chemical Allergens. Journal of Investigative Dermatology 99, 107-S108.

European Commission (2003). Directive 2003/15/EC of the European Parliament and of the Council of 27 February 2003 amending Council Directive 76/768/EEC on the approximation of the laws of the member states relating to cosmetic products. Official Journal of the European Union 2003. L66; 26-35.

Felter, S. P., Ryan, C. A., Basketter, D. A. et al. (2003). Application of the risk assessment paradigm to the induction of allergic contact dermatitis. Regulatory Toxicology and Pharmacology 37, 1-10.

Felter, S. P., Robinson, M. K., Basketter, D. A. and Gerberick, G. F. (2002). A review of the scientific basis for uncertainty factors for use in quantitative risk assessment for the induction of allergic contact dermatitis. Contact Dermatitis 47, 257-266.

Gerberick, G. F., Vassallo, J. D., Foertsch, L. M. et al. (2007). Quantification of chemical peptide reactivity for screening contact allergens: A classification tree lmodel approach. Toxicological Sciences 97, 417-427.

Gildea, L. A., Ryan, C. A., Foertsch, L. M. et al. (2006). Identification of gene expression changes induced by chemical allergens in dendritic cells: opportunities for skin sensitization testing. J. Invest Dermatol. 126, 1813-1822.

Griffiths, C. E., Dearman, R. J., Cumberbatch, M. and Kimber, I. (2005). Cytokines and Langerhans cell mobilisation in mouse and man. Cytokine. 32, 67-70.

Guironnet, G., Dalbiez-Gauthier, C., Rousset, F. et al. (2000). In vitro human $\mathrm{T}$ cell sensitization to haptens by monocytederived dendritic cells. Toxicology in Vitro 14, 517-522.

Hartung, T., Bremer, S., Casati, S. et al. (2004). A modular approach to the ECVAM principles on test validity. Altern. Lab Anim 32, 467-472.

Hauser, C. (1990). Cultured epidermal Langerhans cells activate effector T cells for contact sensitivity 6. J Invest Dermatol. 95, 436-440. 
Jowsey, I. R., Basketter, D. A., Westmoreland, C. and Kimber, I. (2006). A future approach to measuring relative skin sensitising potency: a proposal. Journal of Applied Toxicology 26, 341-350.

Loretz, L. J., Api, A. M., Babcock, L. et al. (2008). Exposure data for cosmetic products: Facial cleanser, hair conditioner, and eye shadow. Food and Chemical Toxicology. In press.

Loretz, L., Api, A. M., Barraj, L. et al. (2006). Exposure data for personal care products: hairspray, spray perfume, liquid foundation, shampoo, body wash, and solid antiperspirant. Food and Chemical Toxicology 44, 2008-2018.

Loretz, L. J., Api, A. M., Barraj, L. M. et al. (2005). Exposure data for cosmetic products: lipstick, body lotion, and face cream. Food and Chemical Toxicology 43, 279-291.

Maxwell, G., Aleksic, M., Aptula, A. et al. (2008). Assuring consumer safety without animal testing: a feasibility case study for skin sensitisation. Altern. Lab. Anim. 36, 557-568.

Maxwell, G. and MacKay, C. (2008). Application of a systems biology approach to skin allergy risk assessment. Altern. Lab. Anim. 36, 521-556.

Natsch, A., Emter, R. and Ellis, G. (2009). Filling the concept with data: integrating data from different in vitro and in silico assays on skin sensitizers to explore the battery approach for animal-free skin sensitization testing. Toxicol. Sci. 107, 106121.

Python, F., Goebel, C. and Aeby, P. (2007). Assessment of the U937 cell line for the detection of contact allergens. Toxicology and Applied Pharmacology 220, 113-124.

Rougier, N., Redziniak, G., Mougin, D. et al. (2000). In vitro evaluation of the sensitization potential of weak contact allergens using langerhans-like dendritic cells and autologous T cells. Toxicology 145, 73-82.

Ryan, C. A., Gildea, L. A., Hulette, B. C. et al. (2004). Gene expression changes in peripheral blood-derived dendritic cells following exposure to a contact allergen. 6. Toxicology Letters 150, 301-316.

Sakaguchi, H., Ashikaga, T., Miyazawa, M. et al. (2009). The relationship between CD86/CD54 expression and THP-1 cell viability in an in vitro skin sensitization test--human cell line activation test (h-CLAT). Cell Biol. Toxicol. 25, 109-126.

Sakaguchi, H., Ashikaga, T., Miyazawa, M. et al. (2006). Development of an in vitro skin sensitization test using human cell lines; human Cell Line Activation Test (h-CLAT). II. An inter-laboratory study of the h-CLAT. Toxicology in Vitro 20, 774-784.

Sens-it-iv (2006). Novel Testing Strategies for In Vitro Assessment of Allergens - project summary. http://www. sens-it-iv. eu/files/start/Sens-it-iv_Summary. pdf.

Smith, C. K. and Hotchkiss, S. A. (2001). Allergic Contact Dermatitis. London, UK: Taylor \& Francis.

Trompezinski, S., Migdal, C., Tailhardat, M. et al. (2008). Characterization of early events involved in human dendritic cell maturation induced by sensitizers: cross talk between MAPK signalling pathways. Toxicol. Appl. Pharmacol. 230, 397406.

Tsuchiya, S., Yamabe, M., Yamaguchi, Y. et al. (1980). Establishment and characterization of a human acute monocytic leukemia cell line (THP-1). Int. J. Cancer 26, 171-176.

Upadhye, M. R. and Maibach, H. I. (1992). Influence of area of application of allergen on sensitization in contact dermatitis. Contact Dermatitis 27, 281-286.

US National Research Council (2007). Toxicity Testing in the Twenty-first Century: A Vision and a Strategy. Committee on Toxicity and Assessment of Environmental Agents National Research Council, Washington, DC.

Vocanson, M., Hennino, A., Cluzel-Tailhardat, M. et al. (2006). CD8+ T cells are effector cells of contact dermatitis to common skin allergens in mice. J. Invest Dermatol. 126, 815-820.

\section{Acknowledgements}

In our effort to develop in vitro skin sensitisation approaches, Colipa is closely collaborating with ECVAM (Hartung et al., 2004) and the research consortium entitled "Novel Testing Strategies for In Vitro Assessment of Allergens" (Acronym: Sens-it-iv) sponsored by the European Union Framework Program 6 (Sens-it-iv, 2006).

\section{Correspondence to}

Gavin Maxwell

Safety and Environmental Assurance Centre

Unilever Colworth

Sharnbrook

Bedford MK44 1LQ, UK

e-mail: Gavin.maxwell@unilever.com 